\title{
Tailored mixing inside a translating droplet
}

\author{
R. Chabreyrie ${ }^{1}$, D. Vainchtein ${ }^{2,3}$, C. Chandre $^{4}$, P. Singh $^{5}$, N. Aubry ${ }^{1}$ \\ 1 Mechanical Engineering Department, Carnegie Mellon University, PA 15213, USA \\ 2 School of Physics, Georgia Institute of Technology, GA 30332, USA \\ ${ }^{3}$ Space Research Institute, Moscow, GSP-7, 117997, Russia \\ ${ }^{4}$ Centre de Physique Théorique, Luminy-case 907, F-13288 Marseille cedex 09, France \\ ${ }^{5}$ Mechanical Engineering Department, New Jersey Institute of Technology, Newark, NJ 07102, USA
}

(Dated: October 28, 2018)

\begin{abstract}
Tailored mixing inside individual droplets could be useful to ensure that reactions within microscopic discrete fluid volumes, which are used as microreactors in "digital microfluidic" applications, take place in a controlled fashion. In this article we consider a translating spherical liquid drop to which we impose a time periodic rigid-body rotation. Such a rotation not only induces mixing via chaotic advection, which operates through the stretching and folding of material lines, but also offers the possibility of tuning the mixing by controlling the location and size of the mixing region. Tuned mixing is achieved by judiciously adjusting the amplitude and frequency of the rotation, which are determined by using a resonance condition and following the evolution of adiabatic invariants. As the size of the mixing region is increased, complete mixing within the drop is obtained.
\end{abstract}

PACS numbers: 47.51.+a, 47.61.Ne, 47.52.+j

\section{INTRODUCTION}

Droplets have been proposed as an alternative to standard fluid-stream microfluidics for lab-on-a-chip applications. This microfluidics approach, also referred to as "digital" because it uses "discrete" fluid volumes (droplets) rather than continuous streams, holds great promise due to the possibility of using single droplets as microreactors [1]. Efficient mixing, however, is needed for reactions to occur, but remains difficult to achieve because the Reynolds number (Re) is usually very small and so the flow is laminar. This issue has recently attracted much attention in the literature. For flows in microchannels, while there are many strategies based on altering the channel geometry, the use of forcing alone (see, e.g., [2, 3, 4, 5, 6]) has also proved to be efficient, especially in the case of low Re [7]. The combination of both geometry alteration and forcing has been explored as well [7, 8, 9, 10]. For droplet-based microfluidics, the forcing alone is the preferred strategy as the deformation of the droplet is difficult to control. In almost all cases, the enhancement of mixing in miniature geometries is based on chaotic advection, the stirring phenomenon that stretches and folds fluid elements thus increasing the interfacial area between the two fluids to be mixed. Chaotic advection inside a liquid drop subjected to a forcing (at low Re) has been studied extensively [11, 12, 13, 14, 15, 16, 17] and was obtained experimentally by means of oscillatory flows [18, 19]. In this letter, we focus on unsteady - yet periodic - forcing.

From a dynamical systems viewpoint, the introduction of a time-dependent perturbation or forcing breaks the invariants (related to the symmetries of the unperturbed system), thus introducing resonances between the natural frequencies of the unperturbed problem and the frequency(-ies) of the forcing. Although such resonances create chaotic regions where mixing occurs, in general, chaotic and regular regions co-exist and unexpected regular sizable pockets persist.

In many situations where it is indeed possible to create chaos, controlling the mixing region(s) remains a challenge. Such a control, however, should be possible since a chaotic system is sensitive to changes in parameter values (as it is to changes in initial conditions). These changes should generically modify the resonances, and thus the location and size of the chaotic regions.

Our general approach along these lines is to consider a bounded three-dimensional (3D) flow, which is the superposition of an integrable flow $\mathbf{v}_{0}$ with at least one invariant and a small time-dependent perturbation $\varepsilon \mathbf{v}_{1}(\mathbf{x}, t)$, $0 \leq \varepsilon \ll 1$. If $\mathbf{v}_{0}$ has only one invariant, the phase space contains two-dimensional tori. In this case, the perturbed flow, $\dot{\mathbf{x}}=\mathbf{v}_{0}(\mathbf{x})+\varepsilon \mathbf{v}_{1}(\mathbf{x}, t)$, has poor mixing properties if the amplitude of the perturbation $\varepsilon$ is small, since twodimensional (2D) tori act as barriers to chaotic diffusion (e.g., [20]). If, on the other hand, $\mathbf{v}_{0}$ has two invariants, trajectories of this integrable flow are all periodic. Most of these periodic orbits are expected to be broken by a generic perturbation $\mathbf{v}_{1}$ with an arbitrarily small amplitude $\varepsilon$. Efficient mixing properties might then be obtained with such perturbed flows. In this work, we consider an axisymmetric integrable flow possessing two invariants, thus possibly offering efficient mixing properties after being perturbed.

While many previous works 11, 12, 21, 22] have shown the existence of chaotic behavior in 3D bounded steady flows, we turn our attention to unsteady flows; the added unsteadiness targets the control of the chaotic behavior through resonance phenomena [17, 23, 24]. Specifically, we seek to create a mixing zone of tunable size which remains localized within a well-defined region of the drop. This should also provide a rationale for the route to complete mixing as the perturbation increases. 


\section{MODEL}

\section{A. Flow equation and assumptions}

We consider a spherical Newtonian drop immersed in an incompressible Newtonian flow in the case where the linear external field is characterized by translational velocity and vorticity vectors, similarly to [12]. As in the latter reference, we assume that the local $R e$ is much smaller than one and that the interfacial tension is sufficiently large for the drop to remain spherical.

The internal velocity field is obtained by solving the Stokes flow problem for both the internal and external flows satisfying the continuity of velocity and tangential stress conditions across the drop surface. In addition, we introduce unsteadiness in the problem by making the vorticity time dependent. In a Cartesian coordinate system translating with the center-of-mass velocity of the drop, and with the $z$ axis in the direction of the translation, the paths of passive marker particles are given by the solution of the non-autonomous dynamical system:

$$
\begin{aligned}
u & =\dot{x}=z x-a(t) \omega_{z} y \\
v & =\dot{y}=z y+a(t)\left(\omega_{z} x-\omega_{x} z\right) \\
w & =\dot{z}=\left(1-2 x^{2}-2 y^{2}-z^{2}\right)+a(t) \omega_{x} y
\end{aligned}
$$

where all lengths and velocities have been nondimensionalized by the drop radius and the magnitude of the translational velocity. Here, the vorticity is defined by $\boldsymbol{\omega}=\left(\omega_{x}, \omega_{y}, \omega_{z}\right)=(1 / \sqrt{2}, 0,1 / \sqrt{2})$, the unitary vector corresponding to the axis of rotation, and $a(t)=\varepsilon / 2(1+\cos \omega t)$, characterized by the frequency $\omega$ and the amplitude $\varepsilon$. In this letter, we consider only small amplitudes, i.e. for $0 \leq \varepsilon \ll 1$. Note that the former equations are identical to those in [12] except that the constant vorticity vector has been replaced by $a(t) \boldsymbol{\omega}$. This can be done by either assuming unsteady vorticity in the external flow field, or by applying a time dependent body force. In practice, this could be realized, e.g., by creating a time dependent swirl motion in the external flow or by applying an electric field that exerts a torque on the drop (e.g., 25] it or work on electrorotation). This flow is the superposition of a Hill's vortex and an unsteady rigid body rotation, and the surface of the drop, $r^{2}=x^{2}+y^{2}+z^{2}=1$, is invariant under flow (1).

\section{B. Integrable case}

We now discuss some features of the unperturbed axisymmetric $(2 \mathrm{D})$ flow $(\varepsilon=0)$. The flow possesses two independent integrals of motion, e.g., the streamfunction $\psi$ and the azimuthal angle $\phi$ :

$$
\psi=1 / 2 \rho^{2}\left(1-r^{2}\right), \quad \phi=\arctan y / x,
$$

where $\rho^{2}=x^{2}+y^{2}$ and $\psi \in[0,1 / 8]$. The streamlines of the unperturbed system are lines of constant $\psi$ and $\phi$,

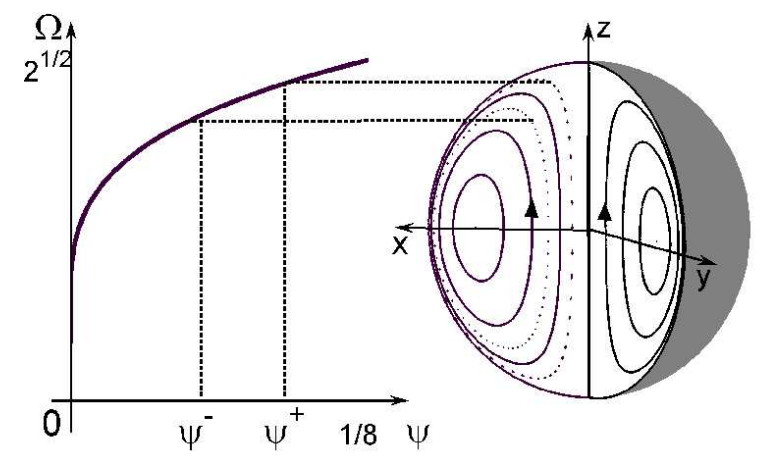

FIG. 1: Streamlines inside the drop (without rotation) and their frequencies $\Omega(\psi)$ as given by Eq. (3).

denoted by $\Gamma_{\psi, \phi}$, and defined as $\left(1-2 \rho^{2}\right)^{2}+(2 \rho z)^{2}=1-$ $8 \psi$ (see Fig. 1). Almost all streamlines are closed curves surrounding a circle of degenerate elliptic fixed points $(\rho=1 / \sqrt{2}, z=0)$. In addition, there are two hyperbolic fixed points located at the poles of the sphere which are connected by heteroclinic orbits. The frequency of the motion on $\Gamma_{\psi, \phi}$ is given by

$\frac{2 \pi}{\Omega(\psi)}=\int_{-\pi / 2}^{\pi / 2} \frac{\sqrt{2} \mathrm{~d} \alpha}{\sqrt{1+\gamma(\psi) \sin \alpha}}=\frac{2 \sqrt{2}}{\sqrt{1+\gamma}} K\left(\sqrt{\frac{2 \gamma}{1+\gamma}}\right)$

where $\gamma(\psi)=\sqrt{1-8 \psi}$ and $K$ is the complete elliptic function of the first kind. The frequency $\Omega$ is bounded by two limits, $\Omega(0)=0$ and $\Omega(1 / 8)=\sqrt{2}$ (see Fig. 11).

On every streamline $\Gamma_{\psi, \phi}$, we introduce a uniform phase $\chi \bmod (2 \pi)$ such that $\chi=0$ on the $x-y$ plane (with $\rho \leq 1 / \sqrt{2}$ ) and $\dot{\chi}=\Omega(\psi)$. The unperturbed system, which can be rewritten in terms of $(\psi, \phi, \chi)$ as

$$
\dot{\psi}=0, \quad \dot{\phi}=0, \quad \dot{\chi}=\Omega(\psi),
$$

belongs to the class of action-action-angle flows.

\section{Perturbed case}

In the perturbed case $0<\varepsilon \ll 1$, the time evolution of the two invariants of the unperturbed system is given by

$$
\begin{aligned}
\dot{\psi} & =-2 a(t) \omega_{x} \psi \sin \phi G(\psi, \chi), \\
\dot{\phi} & =a(t) \omega_{z}-a(t) \omega_{x} \cos \phi G(\psi, \chi),
\end{aligned}
$$

where $G(\psi, \chi)=z / \rho$ is $2 \pi$ periodic in $\chi$ and has zero average in $\chi$. The time evolution equation for $\chi$ is

$$
\dot{\chi}=\Omega(\psi)+a(t) H(\psi, \phi, \chi),
$$

where $H$ is $2 \pi$ periodic in $\chi$. The dynamics possesses two time scales, a fast one (of order one) associated with $\chi$, and a slow one (of order $1 / \varepsilon$ ) associated with $\psi$ and $\phi$. If $\Omega$ and $\omega$ are incommensurate, then the averaging over $\Omega$ and over $\omega$ can be performed independently. In this 
case, the time-periodic terms in Eq. (4) average out, and the averaged system reduces to $\dot{\psi}=0, \quad \dot{\phi}=-\varepsilon / 2$. Thus in the averaged system the value of $\psi$ is conserved as it was in the unperturbed system; in other words, $\psi$ is an invariant of the averaged system. Each trajectory of the averaged system evolves on two-dimensional nested tori $\mathcal{T}_{\psi}$. In the perturbed system, $\psi$ is an adiabatic invariant and the motion follows adiabatically the tori $\mathcal{T}_{\psi}$.

\section{METHODS AND RESULTS}

\section{A. Mixing generation via resonance phenomena}

We now turn to the generation of a 3D chaotic mixing region inside the drop, for which we seek to control both the location and the size. The strategy used for this purpose is to bring a chosen family of unperturbed tori $\mathcal{T}_{\psi}$ into resonance with the perturbation $a(t)$ by adjusting the frequency $\omega$ to satisfy the resonance condition

$$
n \Omega(\psi)-\omega=0,
$$

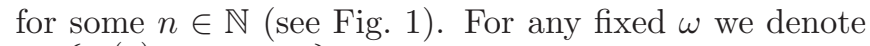
by $\left\{\mathcal{T}^{(n)}(\omega) \mid n \in \mathbb{N}\right\}$ the set of resonant tori $\mathcal{T}_{\psi}$ satisfying (5). Hereafter, we denote the chaotic mixing region generated around $\mathcal{T}^{(1)}(\omega)$ by CMR.

\section{B. Control of the mixing}

Figures 2 and 3 present Liouvillian sections of the perturbed system, which consist of 2D projections of timeperiodic 3D flows by a combination of a stroboscopic map and a Poincaré section (here, the $y=0$ plane). Figure 2 shows that a perturbation $a(t)$ creates a $3 \mathrm{D}$ CMR around $\mathcal{T}^{(1)}(\omega)$ and its location is controlled by varying $\omega$ according to Eq. (5). In what follows, we analyze the location and the size of the CMR as $\omega$ and $\varepsilon$ vary.

For small values of $\omega$, all resonances are located near the pole-to-pole heteroclinic connections (at $\psi=0$, near the $z$ axis and near the boundaries of the drop, see Fig. 2a). As $\omega$ is increased, the CMR penetrates deeper into the drop (Fig. 2b). In the interval $0<\omega<\sqrt{2}$, the CMR is the largest chaotic region (compared to chaotic regions corresponding to higher order resonances), with all the other chaotic regions localized close to the $z$ axis and near the drop boundaries (around the heteroclinic orbits); this is due to the shape of $\Omega(\psi)$. As $\omega$ is increased further, the CMR moves toward the location of the elliptic fixed points of the unperturbed system, closely following the location of the resonant torus $\mathcal{T}^{(1)}(\omega)$ (Fig. 2 c). As the value of $\omega$ approaches $\sqrt{2}$, the CMR shrinks to the circle of elliptic fixed points (Fig. [5).

Whereas the frequency $\omega$ of the rigid body rotation is mostly responsible for the location of the CMR, it is its amplitude $\varepsilon$ which mostly determines its size. Figure 3 shows that the size of the chaotic mixing regions created

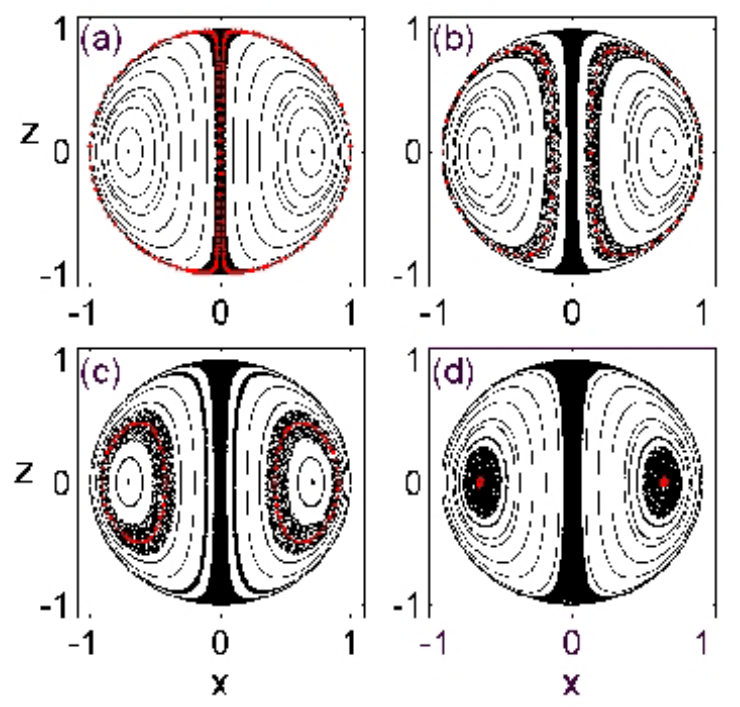

FIG. 2: Liouvillian sections for the amplitude $\varepsilon=0.03$ and the frequencies $\omega=0.55,0.93,1.28,1.41$ (a-d). The (red) dashed line inside the CMR is the torus $\mathcal{T}^{(1)}$.

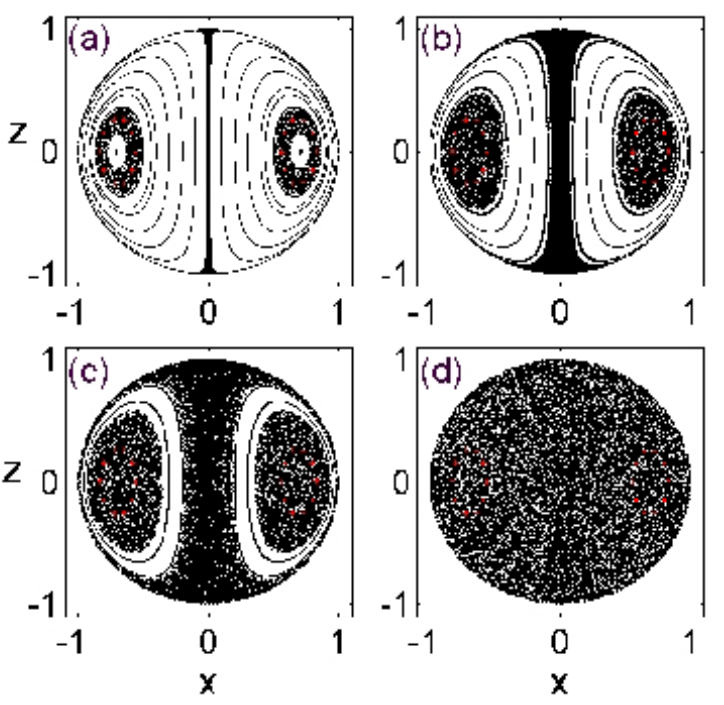

FIG. 3: Liouvillian sections for the frequency $\omega=1.376$ and the amplitudes $\varepsilon=0.01,0.05,0.10,0.20$ (a-d).

by the $n=1$ resonance and by higher order resonances (mostly the $n=2$ resonance) increases as the amplitude of the perturbation increases. Around $\varepsilon \approx 0.20$, the chaotic regions around the heteroclinic orbits and the CMR join together to cover the entire drop volume.

Recall that in the averaged system the adiabatic invariant $\psi$ is constant. In the exact system, however, along a given trajectory starting at $\psi=\psi_{0}$ it varies between $\psi^{-}\left(\psi_{0} ; \omega, \varepsilon\right)$ and $\psi^{+}\left(\psi_{0} ; \omega, \varepsilon\right)$. The width $\Delta \psi=$ $\psi^{+}\left(\psi_{0} ; \omega, \varepsilon\right)-\psi^{-}\left(\psi_{0} ; \omega, \varepsilon\right)$ is small away from the resonance, and increases significantly closer to the resonance. The projection of three characteristic trajectories onto the $(\psi, \phi)$-plane (called the slow plane in dynamical sys- 

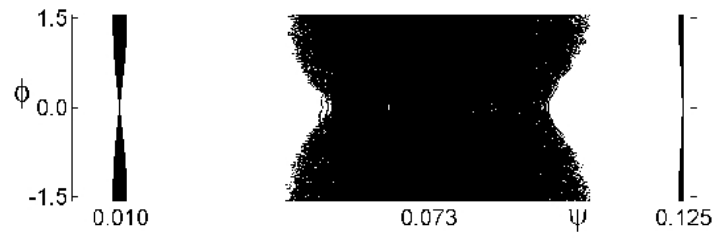

FIG. 4: Projection of three characteristic trajectories on the slow phase plane, with $\phi_{0}=0$ and $\psi_{0}=0.010,0.073,0.125$.
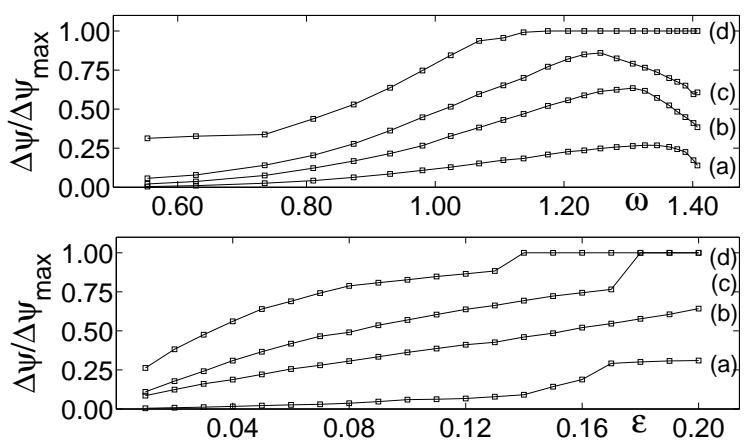

FIG. 5: Size of the chaotic mixing region; Upper panel: Normalized $\Delta \psi v s$. $\omega$ for the amplitudes $\varepsilon=0.01,0.05,0.10,0.20$ (a-d); Lower panel: Normalized $\Delta \psi$ vs. $\varepsilon$ for $\omega=$ $0.55,0.93,1.28,1.41$ (a-d).

tems) is presented in Fig. 4. The narrow regions on the sides are off-resonance trajectories that stay quite close to the corresponding tori $\mathcal{T}_{\psi}$. In between, the middle trajectory deviates much further from its $\mathcal{T}_{\psi}=\mathcal{T}^{(1)}(\omega)$ and fills the entire CMR. The quantity $\Delta \psi$ is probably the most convenient quantity to estimate the size of the CMR (around $\mathcal{T}^{(1)}(\omega)$ for $\omega<\sqrt{2}$ ). The volume between the tori $\mathcal{T}_{\psi^{-}}(\omega)$ and $\mathcal{T}_{\psi^{+}}(\omega)$ gives the CMR size in $3 \mathrm{D}$. The dependence of the size of the CMR (in terms of $\Delta \psi$ ) on $\varepsilon$ and $\omega$ is illustrated in Fig. 5. The curves (a)-(d) in the upper and lower panels correspond to the Figs. 2 $a-d$ and $[3 \mathrm{a}-\mathrm{d}$, respectively. For a given $\omega$ value (i.e. for a given $\mathcal{T}^{(1)}(\omega)$ ), the size can be controlled by adjusting the value of $\varepsilon$; for example in the range of frequencies $1.181 \leq \omega \leq 1.357$, the entire droplet exhibits chaotic mixing for $\varepsilon \geq 0.175$. For each smaller value of $\varepsilon$ the size reaches a maximum for a certain value $\omega^{m}(\varepsilon)$ of the frequency. On the one hand, this property can be used as an optimization technique to obtain the maxi- mal CMR size one can reach for a given amplitude $\varepsilon$ of the rotation. On the other hand, $\Delta \psi$ versus $\varepsilon$ increases quite monotonically for all values of $\omega$. The derivation of the maxima locations and estimates of $\Delta \psi$ as functions of the parameters and the order of resonance, will be addressed elsewhere. The structure of the CMR in our case is rather different from that obtained in other problems that possess resonance-induced chaotic advection. Namely, here the size of the CMR vanishes as $\varepsilon$ goes to 0 and the CMR is localized near the resonance. In contrast, in the flow considered in, e.g., [17], the mixing is caused by resonances, but the CMR occupies a volume on the scale of the whole system. The difference comes from the fact that the averaged change of the frequency of the fast system vanishes in the current system, thus preventing the trajectories starting away from the resonance from approaching it. This property makes the kind of flows investigated here useful as it may be advantageous to localize the mixing in certain parts of the system only.

\section{CONCLUSION}

In summary, we have shown that by applying a judicious oscillatory rotation to a translating drop (an integrable system), one can create a chaotic mixing zone with a prescribed location and size. The appropriate values of the parameters of the perturbation (here, a rotation of a given frequency and amplitude) are determined by quantitative features of the integrable case. For any amplitude of the rotation, the frequency optimizing the CMR size has been obtained. Such an optimization could be useful in guiding the design of practical mixing devices aiming at the best possible mixing rate within individual drops.

\section{Acknowledgments}

This article is based upon work partially supported by the NSF (grants CTS-0626070 (N.A.), CTS-0626123 (P.S.) and 0400370 (D.V.)). D.V. is grateful to the RBRF (grant 06-01-00117) and to the Donors of the ACS Petroleum Research Fund. C.C. acknowledges support from Euratom-CEA (contract EUR 344-88-1 FUA F).
[1] H. Song, J.D. Tice and R. F. Ismagilov, Angew. Chem. int. Ed. 42, 768 (2003).

[2] M.H. Oddy, J.G Santiago, J.C. Mikkelsen, Anal. Chem. 73, 5822 (2001).

[3] H.H. Bau, J. Zhong and M. Yi, Sensors and Actuators B 79, 207 (2001).

[4] A. Ould El Moctar, N. Aubry and J. Batton, Lab Chip 3, 273 (2003).
[5] I.K. Glasgow and N. Aubry, Lab Chip 3, 114 (2003).

[6] I.K. Glasgow, J. Batton and N. Aubry, Lab Chip 4, 558 (2004).

[7] A. Goullet, I.K. Glasgow and N. Aubry, Mech. Res. Commun. 33, 739 (2006).

[8] X. Niu and Y-K. Lee, J. Micromech. Microeng. 13, 454 (2003).

[9] F. Bottausci et al., Phil. Trans. Royal Soc. A 362, 1001 
(2004).

[10] M.A. Stremler, F.R. Haselton and H. Aref, Phil. Trans. Royal Soc. A 362, 1019 (2004).

[11] K. Bajer and H.K. Moffatt, J. Fluid Mech. 212, 337 (1990).

[12] D. Kroujiline and H.A. Stone, Physica D 130, 105 (1999).

[13] S.M. Lee, D.J. Kim and I.S. Kang, Phys. Fluids 12, 1899 (2000).

[14] T. Ward and G.M. Homsy, Phys. Fluids 13, 3521 (2001).

[15] R.O. Grigoriev, Phys. Fluids 17, 033601 (2005).

[16] X.M. Xu and G.M. Homsy, Phys. Fluids 19, 013102 (2007).

[17] D. Vainchtein, J. Widloski and R. Grigoriev, Phys. Rev. Lett. 99, 094501 (2007).
[18] T. Ward and G.M. Homsy, Phys. Fluids 15, 2987 (2003).

[19] R.O. Grigoriev, M.F. Schatz and V. Sharma, Lab Chip, 6, 1369 (2006).

[20] M. Feingold, L. Kadanoff and O. Piro, J. Stat. Phys. 50, 529 (1988).

[21] D. Vainchtein, A. Vasiliev and A. Neishtadt, Chaos 6, 67 (1996).

[22] D. Vainchtein, A. Neishtadt and I. Mezić, Chaos 16, 043123 (2006).

[23] R. Lima and M. Pettini, Phys. Rev. A 41, 726 (1990).

[24] J.H.E. Cartwright, M. Feingold and O. Piro, J. of Fluid Mech. 316, 259 (1996).

[25] N. Aubry and P. Singh, Electrophoresis 27, 703 (2006). 